Technical Paper

\title{
Feedforward control of sheet bending based on force measurements
}

\author{
Jos Havinga ${ }^{\mathrm{a}, *}$, Ton van den Boogaard ${ }^{\mathrm{a}}$, Franz Dallinger ${ }^{\mathrm{b}}$, Pavel Hora ${ }^{\mathrm{b}}$ \\ ${ }^{a}$ Faculty of Engineering Technology, University of Twente, Drienerlolaan 5, Enschede, The Netherlands \\ ${ }^{\mathrm{b}}$ Institute of Virtual Manufacturing, ETH Zürich, Tannenstrasse 3, Zürich, Switzerland
}

\section{A R T I C L E I N F O}

\section{Article history:}

Received 2 December 2016

Received in revised form 4 October 2017

Accepted 6 October 2017

\section{Keywords:}

Metal forming

Sheet bending

Product-to-product variation

Feedforward control

Force measurement

Process estimator

LASSO regression

\begin{abstract}
A B S T R A C T
The accuracy which can be reached with feedback control in industrial metal forming processes is limited by product-to-product variations in the process. These variations may be controlled with a feedforward control system. To do so, a measurement system and a process estimator are needed to measure and correct for these variations. In this work, the use of force measurements for feedforward control of a steel sheet bending process is investigated. A multi-stage demonstrator process is developed with cutting, deep drawing and bending stages and a large number of force sensors. Several relations between process forces and the product geometry are studied in an extensive analysis of measurement data from the production process. It is proposed to build a moving window process estimator using the LASSO regression method. The parameters of the regression model are updated during production based on historical data of the production line. Several simulation runs are performed to estimate the effect of feedforward control on the process accuracy. These simulation runs are based on measurements from the real production line and minor assumptions. The proposed approach leads to an estimated decrease of $24 \%$ in the root mean square error of the final angle with respect to a simulation run with feedback control only.
\end{abstract}

(c) 2017 The Society of Manufacturing Engineers. Published by Elsevier Ltd. All rights reserved.

\section{Introduction: control of metal forming processes}

Metal forming processes suffer from variations of material properties and process conditions. These disturbances affect the final properties of the product. For products with narrow tolerances, closed-loop control may be used to ensure product quality. A driving factor for recent developments in control of forming processes is the use of information technology, enabling for real-time processing of increasingly detailed sensor data and process models [1].

An extensive review on control of metal forming processes is given in the work of Polyblank et al. [2]. They observed that control systems in forming processes are usually designed to control the movement of the tooling, but lack the ability to control the final state of the product, such as its geometry or stress distribution. On the other hand, they foresee an expansion in the applications of closed-loop control in metal forming, based on the accessibility of the main components needed for process control: sensors, actuators and process models. Given the ongoing development of computing power, increasingly detailed process models and con-

\footnotetext{
* Corresponding author.

E-mail address: joshavinga@gmail.nl (J. Havinga).
}

trol systems may be developed in pursuit of a higher accuracy of forming processes.

An important factor for the design of manufacturing control systems is the rate of change of the process disturbances. Disturbances which change slowly over time may be controlled with feedback systems, adapting the process settings based on measurements from finished products. However, product-to-product variations cannot be eliminated with such systems and must be controlled with feedforward control based on measurements from the semifinished products. In the current work, the eligibility of the use of force measurements in a feedforward estimator of a sheet bending process is investigated. The question is whether the variations in the process are reflected by the force measurements. Several researchers have investigated the use of force measurements in control of forming processes (Section 2). Different methods have been developed to adjust for changes in production conditions (e.g. changes in nominal sheet thickness or in used material). However, little work has been published on control of product-to-product variations in metal forming processes. This is reflected by the small number of products used for training of control models and for validation of the control methods: for most studies the dataset size does not exceed 100 products (Table 1 ).

To study product-to-product variations in metal forming, large data sets with measurements from every product in the production line are needed. To the best of our knowledge, no studies 
Table 1

Studies on control of forming processes which relate force measurements to final product properties and include experimental results.

\begin{tabular}{|c|c|c|c|c|c|}
\hline \multirow[t]{2}{*}{ Publication } & \multirow[t]{2}{*}{ Process } & \multirow[t]{2}{*}{ Model } & \multirow[t]{2}{*}{ Variation in process conditions } & \multicolumn{2}{|c|}{ Number of products } \\
\hline & & & & Training set & Validation tests \\
\hline Hardt et al. [4] & Roll straightening & Linear analytical & Two materials & $\mathrm{n} / \mathrm{a}$ & 8 \\
\hline Müller-Duysing [5] & Air bending & Linear regression & $\begin{array}{l}\text { Three materials, multiple } \\
\text { thicknesses and cycle times }\end{array}$ & Unclear & 44 \\
\hline Yang et al. [6] & Air bending & $\begin{array}{l}\text { Fuzzy model with experimental } \\
\text { data }\end{array}$ & Seven lots of one material & Unclear & Unclear \\
\hline Forcellese et al. [7] & Air bending & $\begin{array}{l}\text { Neural network with experimental } \\
\text { data }\end{array}$ & $\begin{array}{l}\text { Three thicknesses with two lots } \\
\text { each }\end{array}$ & $30 / 60$ & 84 \\
\hline $\begin{array}{l}\text { Nastran and } \\
\text { Kuzman [8] }\end{array}$ & Wire straightening and bending & $\begin{array}{l}\text { Numerical model linearized } \\
\text { around operating point }\end{array}$ & No & $\mathrm{n} / \mathrm{a}$ & Large number \\
\hline $\begin{array}{l}\text { Viswanathan et al. } \\
\text { [9] }\end{array}$ & Channel forming & $\begin{array}{l}\text { Neural network with experimental } \\
\text { data }\end{array}$ & $\begin{array}{l}\text { Six materials, varying thicknesses } \\
\text { in between } 0.70 \text { and } 0.85 \mathrm{~mm} \text { and } \\
\text { five lubrication conditions }\end{array}$ & 80 & 12 \\
\hline Heller et al. [10] & Air bending & $\begin{array}{l}\text { Linear model with semi-analytical } \\
\text { simulation data }\end{array}$ & $\begin{array}{l}\text { Three materials, three thicknesses } \\
\text { and four target angles }\end{array}$ & $\mathrm{n} / \mathrm{a}$ & 72 \\
\hline $\begin{array}{l}\text { Longo and } \\
\text { Maccarini [11] }\end{array}$ & Air bending & $\begin{array}{l}\text { Linear interpolation with } \\
\text { simulation data }\end{array}$ & No & $\mathrm{n} / \mathrm{a}$ & 5 \\
\hline Groche et al. [12] & Air bending & Linear regression & $\begin{array}{l}\text { Three materials, three thicknesses } \\
\text { and three target angles }\end{array}$ & Unclear & 30 \\
\hline
\end{tabular}

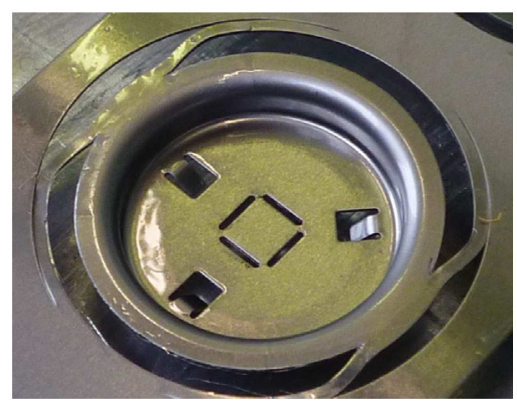

Fig. 1. Demonstrator product.

with an extensive analysis of a large number of measurements have been presented in the literature on metal forming processes. In the MEGaFiT project [3], a metal forming demonstrator process has been developed to investigate the feasibility of control of product-to-product variations in metal forming. The demonstrator process includes deep drawing, forging and bending steps. In this work, the use of force measurements for control of the bending stage is investigated. Section 2 is a literature study on the use of force measurements for control of metal forming processes. In Section 3 the demonstrator process is presented. In Section 4 several correlations between measurement data are shown to deepen the insight into the demonstrator process. In Section 5 a proposal is made for a model based on experimental data to be used in the control of the bending process. A series of simulation runs are performed using the proposed method in combination with measurement data obtained from the demonstrator process in order to assess the potential effect of a feedforward control system based on force measurements. Finally, conclusions and discussion are found in Section 6.

\section{Literature: force measurements for feedforward control}

The following overview is limited to studies with experimental data on the relations between force measurements and product properties, whereas purely numerical studies are neglected. A summary of the discussed papers is given in Table 1.

Hardt et al. [4] implemented force and curvature measurements in a roll straightening process. They estimated the curvature after unloading based on a user-defined value of the elastic bending stiffness and force and curvature measurements. In a set of eight experiments, they achieved a reduction of 53-95\% for the maximum lateral deflection of the product.

Müller-Duysing [5] developed a control system for air bending based on measurement of the intermediate angle and the force during bending. The intermediate angle at three different positions of the punch and the bending force at two different positions of the punch were used as input of a linear regression model. After optimizing the regression coefficients based on measured data, the Root-Mean-Square Error (RMSE) of the prediction error of the model was $0.17^{\circ}$ over a set of 44 products with three different materials. The RMSE of the prediction error of the best model without force measurement was $0.24^{\circ}$.

Yang et al. [6] created an experimental database of an air bending process with force and angle measurements and other information such as sheet thickness, ambient temperature and material name. For the control of a new workpiece, data from the database was selected based on a fuzzy logic system. Using this selection, they compared the experimental force curves with the measured force curve and determined the maximum force needed to achieve the desired angle. An accuracy of $0.1^{\circ}$ was reached when the database was filled with information similar to that of the processed workpiece. An accuracy of $0.25^{\circ}$ was reached when a database with a large variety of experimental data was used in combination with the modified fuzzy models. The uncontrolled accuracy is not mentioned in the paper.

A study on air bending with three different sheet thicknesses was performed by Forcellese et al. [7]. They fitted the force curve to a regression model with five parameters and related the parameters to the final angle of the strip using neural network models. They studied the effect of the training set size of the neural network models on the bending accuracy and found standard deviations in the range of $0.11-0.23^{\circ}$ for the angle with the different training set sizes and thicknesses. The uncontrolled accuracy is not reported.

Nastran and Kuzman [8] examined a wire bending process which is preceded by a straightening process. They observed a strong correlation between the force on the rollers during straightening and the final geometry after bending. They developed a model to relate the variation of the force to variation of the yield stress and proposed to control the position of the rollers to affect the amount of plastic work during straightening and keep the yield stress of the incoming wire for the bending process at a constant level.

Control of a steel channel forming process by adjusting the binder force trajectory was investigated by Viswanathan et al. [9]. The coefficients of a third order polynomial fit of the force curve 


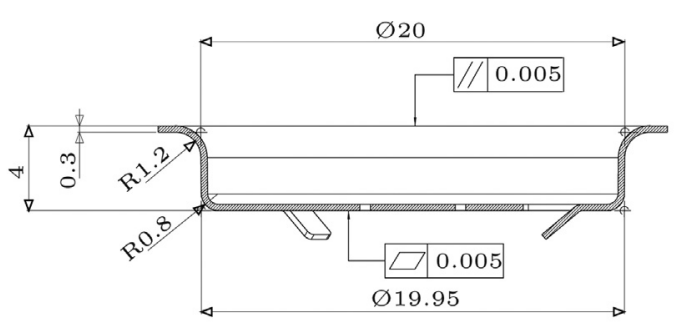

A-A



Fig. 2. Technical drawing of demonstrator product, including numbering of the flaps. The forged profile in the center of the cup is not drawn.

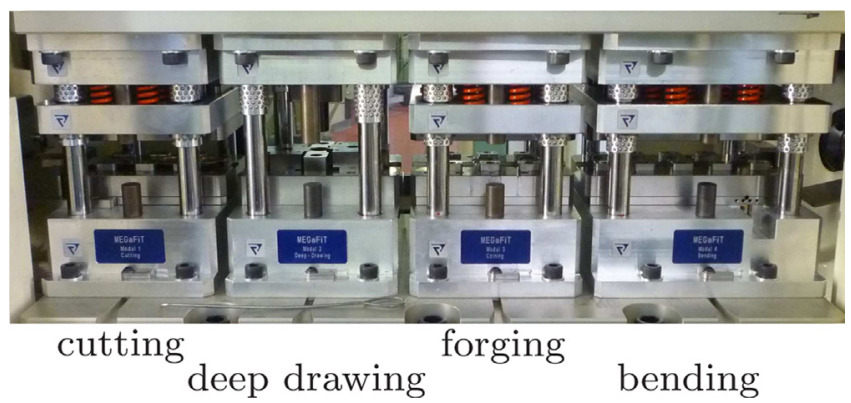

Fig. 3. Tooling.

were used as input of a two-layer neural network. After training the network with five different steel sheets and four different lubrication conditions, it was used to control the springback of the product. New tests were performed with the same steel grades as used for training the neural network. Springback values within specifications $\left(10^{\circ}-12^{\circ}\right)$ were found. However, two out of the four experiments with other steel grades resulted in products out of specification.

Heller et al. [10] used force and angle measurements in an air bending process with partial unloading. Using simulation data and a linear relation between the measured force at the fully loaded and the partially unloaded state, they determined the maximum force needed to achieve the desired angle. In duplicate experiments with three different materials, three different sheet thicknesses and four different target angles, they found an average error of $0.4^{\circ}$.

Longo and Maccarini [11] used an finite element model to estimate material properties based on the force curve in air bending. The estimate was used to predict the angle after springback. They found good agreement in the average predicted springback angle over a set of five experiments.

Groche et al. [12] used the maximum blanking force from a previous process stage and the sheet thickness to predict the angle after springback in air bending, based on a linear regression model built with historical data from the process. The feedforward control loop was combined with a feedback controller and the approach showed improved performance with respect to the use of feedback control only.

The significance of force measurements for control of metal forming processes is evidently shown throughout the abovementioned studies. However, force measurements have been mainly used to adapt the manufacturing process to changes in process conditions. In this work, the use of force measurements for predicting product-to-product variations is studied.

\section{Demonstrator process}

A demonstrator process has been designed to investigate the feasibility of product-to-product control in metal forming. The demonstrator product is shown in Fig. 1. Three types of forming processes have been used in the process: deep drawing, forging and bending. The product is produced in an industrial environment at a production rate of one product per second. It is formed from a 280 or $300 \mu \mathrm{m}$ thick and $38 \mathrm{~mm}$ wide stainless steel strip and it remains attached to the strip during all process steps. The main dimensions of the product are shown in Fig. 2.

The tooling consists of four modules: cutting, deep drawing, forging and bending (Fig. 3), mounted on an industrial stamping press. Every module has several positions in which the forming steps are performed. The order and numbering of the process steps is shown in Fig. 4. During each stroke of the press all products move one position. Therefore, the total production time of one product is around twenty seconds. The strip remains aligned in the tooling by the use of pilot holes and pins.

The first module has three cutting stages. The second module has a single deep drawing stage, with four blank holder sections which are controlled independently with a hydraulic system. This enables control of the roundness of the flange, which is influenced by the anisotropy of the material. The hydraulic system is automatically re-pressurized to the maximum pressure $p_{\max }$ when its pressure drops below the minimum pressure $p_{\min }$. The third module has two cutting stages and one forging stage to form six $200 \mu \mathrm{m}$ wide parallel ribs in the bottom of the cup. The height of the ribs depends on the applied forging force [13].

In the last module, three flaps are cut out in the bottom of the cup. Thereafter, the flaps are bent to $50^{\circ}$ in a closed die (Fig. $5 \mathrm{a}$ ), and finally they are bent back to a desired angle in an open bending stage (Fig. 5b). During the first bending stage, the bending forces of all flaps are measured. The force-displacement curve from this bending stage is strongly nonlinear due to the geometry of the tooling. The bending force measurements and the interpretations thereof are discussed in Section 4.3. In the second bending stage, the amount of back bending can be adapted from product to product. As shown in Fig. 5b, a horizontal translation which is imposed by a servo motor converts into vertical movement of the back bending punch through a slanted groove with a ratio of 1 to 10 . The actuator does not move during contact between the punch and the product. In the last stage of the bending module, a picture of the side of flap 2 is taken to determine the angle (Fig. 6). The standard deviation of the angle measurement error is determined to be less than $0.01^{\circ}$.

A large amount of data is stored during manufacturing. Process forces, blank holder pressure, flap angle and incoming sheet thickness are measured and stored during production. An overview of the sensors is given in Table 2. Relevant information is obtained 


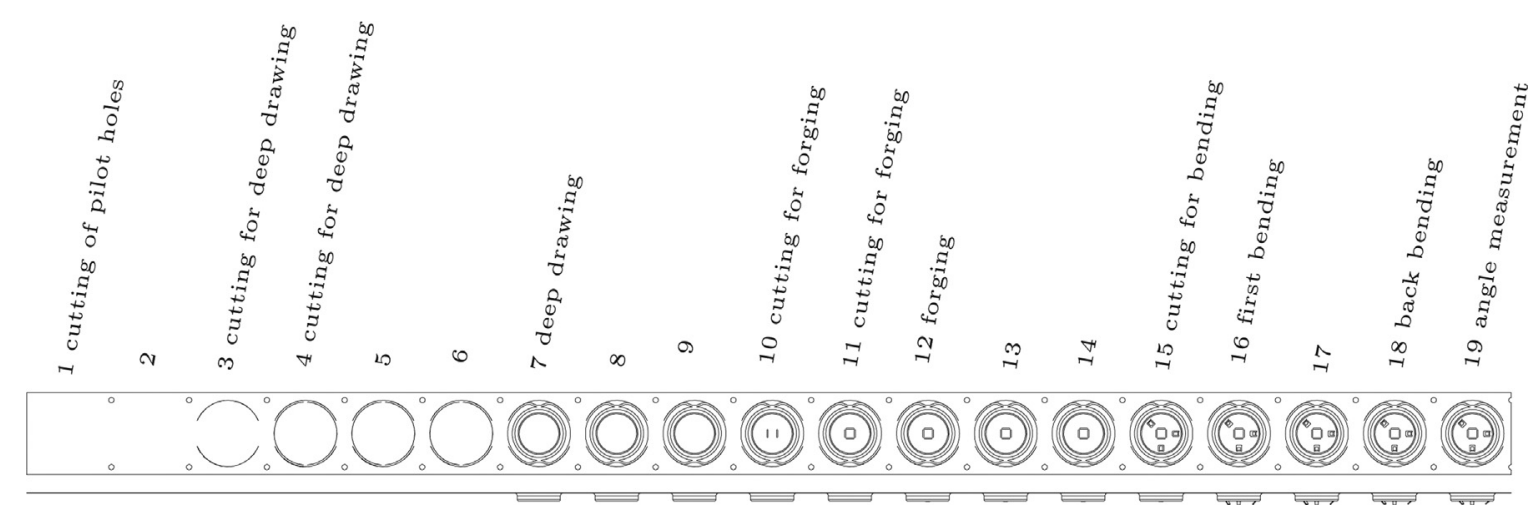

Fig. 4. Production steps of demonstrator product.

Table 2

Overview of sensors in the demonstrator process.

\begin{tabular}{|c|c|c|c|c|c|}
\hline Process stage (nr) & Description & Sensor & Range & Sampling rate & Amount \\
\hline & Thickness & Vollmer & $0-1 \mathrm{~mm}$ & & 1 \\
\hline Cutting (1) & Pilot hole cutting force & Kistler SlimLine 9132B29 & $0-7 \mathrm{kN}$ & $5 \mathrm{kHz}$ & 1 \\
\hline Deep drawing (7) & Punch force & Kistler SlimLine 9133B29 & $0-14 \mathrm{kN}$ & $5 \mathrm{kHz}$ & 1 \\
\hline Deep drawing (7) & Blank holder pressure & & & $5 \mathrm{kHz}$ & 4 \\
\hline Forging (12) & Punch force & Kistler Slimline 9134B29 & $0-26 \mathrm{kN}$ & $5 \mathrm{kHz}$ & 1 \\
\hline Bending (16) & Punch force & Kistler SlimLine 9130B39 & $0-3 \mathrm{kN}$ & $5 \mathrm{kHz}$ & 3 \\
\hline Bending (19) & Flap angle & AVT Stingray CCD & $20-80^{\circ}$ & & 1 \\
\hline
\end{tabular}

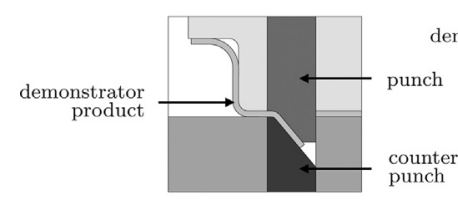

(a)

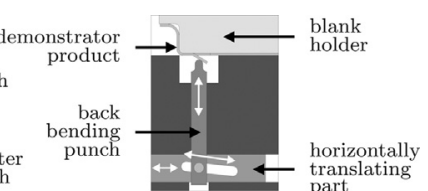

(b)

Fig. 5. The two forming steps from the bending module. (a) Over bending stage (stage 16). (b) Back bending stage (stage 18).

from the sensors during the fraction of the stroke where there is contact between the tooling and the product, whereas no signal is measured during the rest of the stroke. The number of relevant sampling points for each process stage depends on the sensor sampling rate, the exact geometry of the tooling, the stamping rate (1 stoke per second) and the total stroke length of the press ( $38 \mathrm{~mm})$. The number of sampling points during contact between tooling and product is roughly 100 for the cutting stage, 700 for the deepdrawing stage, 350 for the forging stage and 450 for the bending stage. In each test, all raw data are stored and post-processed with offline procedures such as filtering and aligning of force measurements. More details on the post-processing can be found in Havinga [14]. An overview of post-processed sensor signals can be found in Fig. 7.

Four tests with different settings were performed. An overview of the tests is given in Table 3. At least 3000 products have been produced in every test. Two different nominal sheet thicknesses have been used. The values $p_{\min }$ and $p_{\max }$ denote the lower and upper bound for the pressure of the hydraulic system for the deep drawing blank holders. For every sheet thickness, one test with and one test without back bending was performed. Some changes have been made to the tooling for test number 4 . The purpose of these changes was to minimize the disturbances to the process caused by the tooling. The stability of the hydraulic system of the deep drawing stage is poor, as will be discussed in Section 4.4. The stability was improved by reducing the bandwidth of the pressure of the hydraulic system. Furthermore, the forging punch was removed to decrease the loads on the tooling.

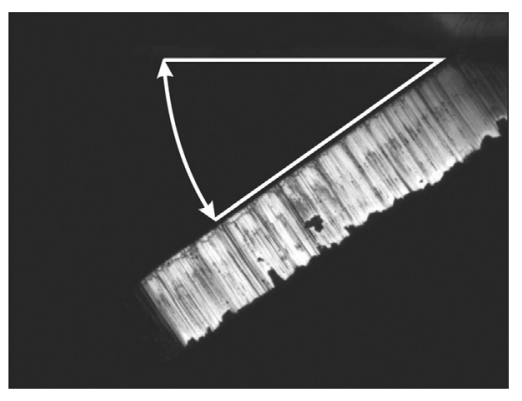

Fig. 6. Picture of flap from inline camera (step 19).

\section{Test results}

Several long test runs have been performed with the demonstrator process. All force measurements during deformation of the product are stored for all products. Hence, thousands of data points are stored per product.

The objective of this work is to decrease product-to-product variation in the demonstrator process using feedforward control based on force measurements. To do so, the relations between force measurements and the final angle of the flap have to be identified. In Section 5, this is done with regression analysis. However, it is hard to gain insight about the measurements based on these regression models only. The measurements and their relations with the final angle of the flap are the key factor for feedforward control of the demonstrator process. Therefore, several observations about the measurements are discussed in this section.

This section starts with the definition of a scaled correlation measure in Section 4.1. This measure is used for analysis of the data. Thereafter, the following aspects of the data are discussed: the amount of variation of the angle (Section 4.2), the bending forces (Section 4.3), variation in the deep drawing stage (Section 4.4), the effect of thickness variation (Section 4.5) and the correlation between the forces and the angle (Section 4.6). 
Table 3

Overview of datasets.

\begin{tabular}{|c|c|c|c|c|}
\hline Dataset number & Nominal thickness & Back bending & Blankholder $p_{\min } / p_{\max }$ & Dataset size \\
\hline 1 & $280 \mu \mathrm{m}$ & Yes & 100/130 bar & 3507 products \\
\hline 2 & $280 \mu \mathrm{m}$ & No & 100/130 bar & 3268 products \\
\hline 3 & $300 \mu \mathrm{m}$ & Yes & 100/130 bar & 3142 products \\
\hline 4 & $300 \mu \mathrm{m}$ & No & 100/105 bar & 3530 products \\
\hline
\end{tabular}

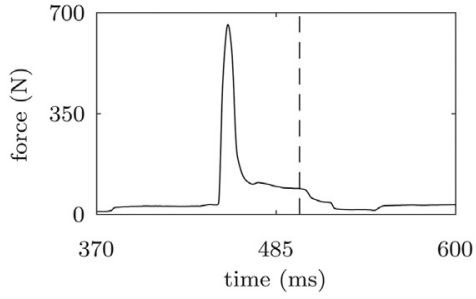

(a)

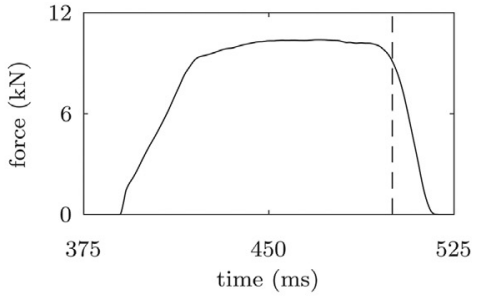

(b)

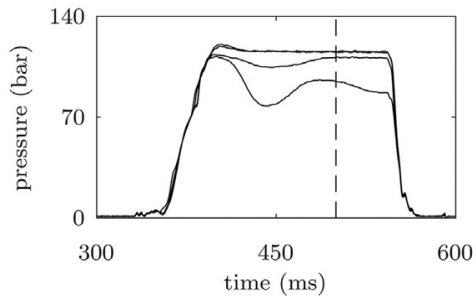

(c)

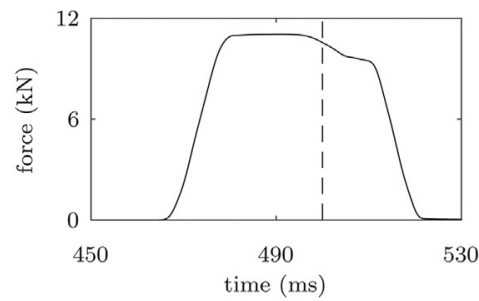

(d)

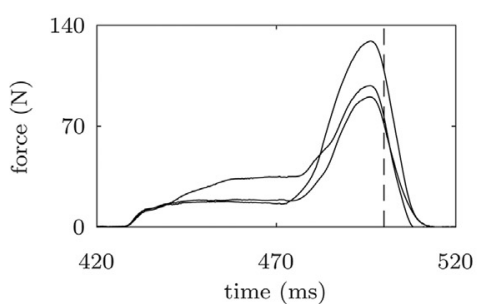

(e)

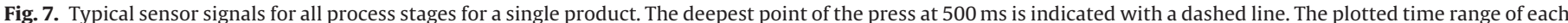

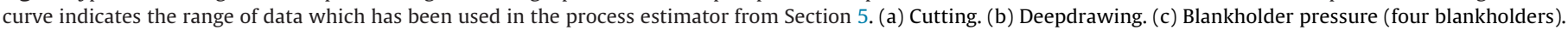
(d) Forging. (e) Bending (three punches).

\subsection{Scaled correlation}

One of the tools for identifying correlations between two signals is the cross-correlation plot. In the case of control of metal forming processes, the long-term correlation is of no interest, because longterm variations can be controlled with feedback control. Therefore, a scaled correlation measure is used to determine the short-term correlation [15]. The scaled correlation coefficient is based on the Pearson correlation coefficient. The Pearson correlation between two signals $X$ and $Y$ is:

$\rho(X, Y)=\frac{\operatorname{cov}(X, Y)}{\sigma_{X} \sigma_{Y}}$

The covariance between $X$ and $Y$ is $\operatorname{cov}(X, Y)$, and the standard deviations of $X$ and $Y$ are $\sigma_{X}$ and $\sigma_{Y}$ respectively. For the demonstrator process, $X$ and $Y$ may be measurements such as the thickness or the maximum bending force for each product.

The scaled correlation factor is determined as follows: a bin width $n_{b}$ is chosen, and the complete dataset is divided into $K$ non-overlapping bins with a width of $n_{b}$ products. The Pearson correlation coefficient is determined in every bin, and the scaled correlation coefficient $\rho_{s}$ is defined as the average of these values:

$\rho_{s}=\frac{1}{K} \sum_{i=1}^{K} \rho_{i}$

where $K$ is the integer part of the number of products in the dataset divided by the bin width $n_{b}$. In this work, a bin width $n_{b}$ of 10 products is used.

The scaled correlation factors are used in cross-correlation plots. Cross-correlation is defined as:

$\rho_{s}(\tau)=\rho_{s}(X(\boldsymbol{t}), Y(\boldsymbol{t}+\tau))$
The factor $\tau$ is a shift in the numbering of the products. If - as an example - the dataset size is 1003 products, $X$ is the thickness and $Y$ is the maximum bending force, $\rho_{s}(3)$ is the scaled correlation between the thickness of products 1 to 1000 and the maximum bending force of products 4 to 1003. In the case of auto-correlation, $X$ and $Y$ are equal.

\subsection{Angle variation}

The measured angles from the different tests are shown in Fig. 8. In each plot, the scaling of the horizontal axis in between products number 1000 and 1050 is changed, to give an impression of the amount of product-to-product variation. Product-to-product variation represents a significant part of the total variation in all tests. The tests with back bending (Fig. 8a and c) show more variation than the tests without back bending (Fig. 8b and d). Sudden jumps in the angle are observed in the tests with back bending. However, this does not imply that the jumps are caused by disturbances from the back bending stage. In Section 4.6 it will be shown that some of these jumps can be predicted with the forces from the over bending stage.

\subsection{Bending force}

In Fig. 9, the bending forces of dataset 3 are shown for all three flaps. The differences between the average force curves per flap are related to the exact tooling geometry and alignment at each bender. The shape of the bending force curve has been studied with a finite element model. During the first part of the stroke, free bending occurs. At around $485 \mathrm{~ms}$, the slope of the force curve increases because the contact area between the punch and the flap starts moving. A second increase of the slope is observed after $490 \mathrm{~ms}$, when the tip of the flap touches the die. The force reaches its max- 


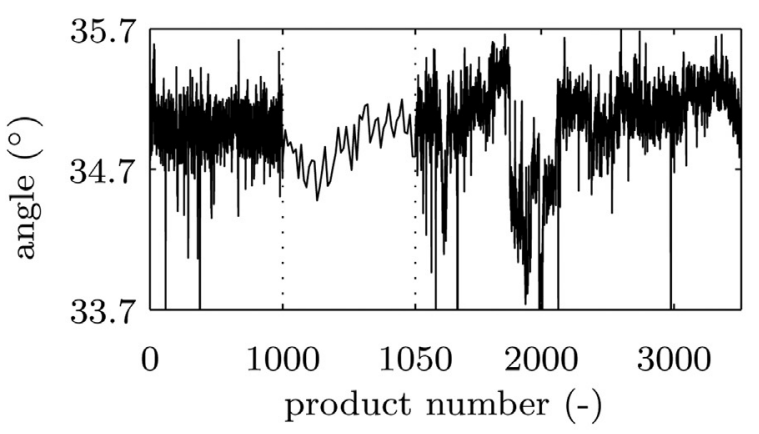

(a)

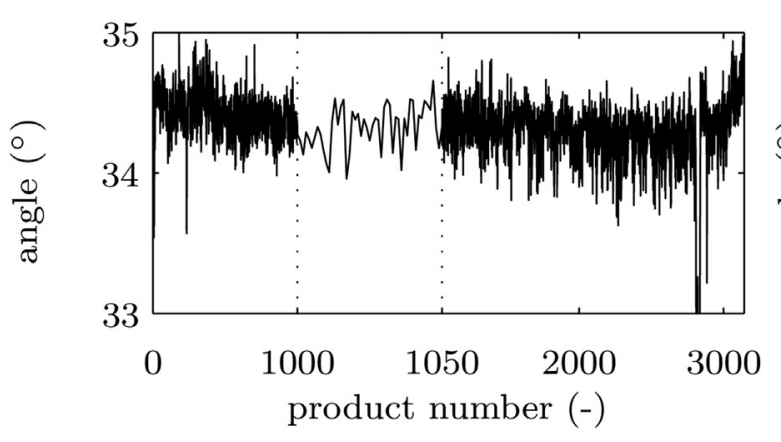

(c)

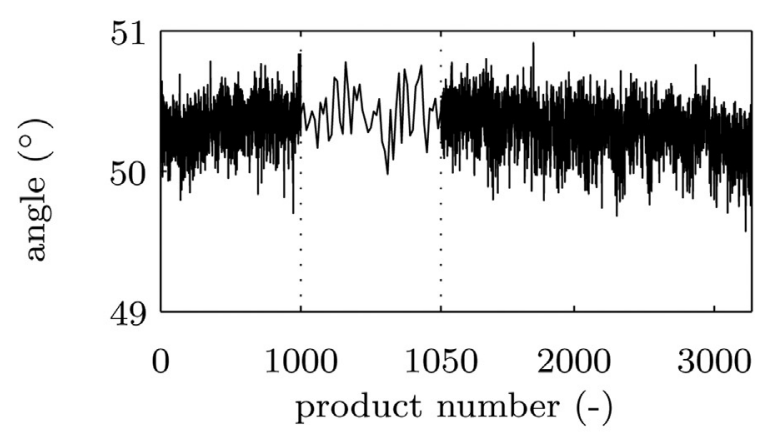

(b)

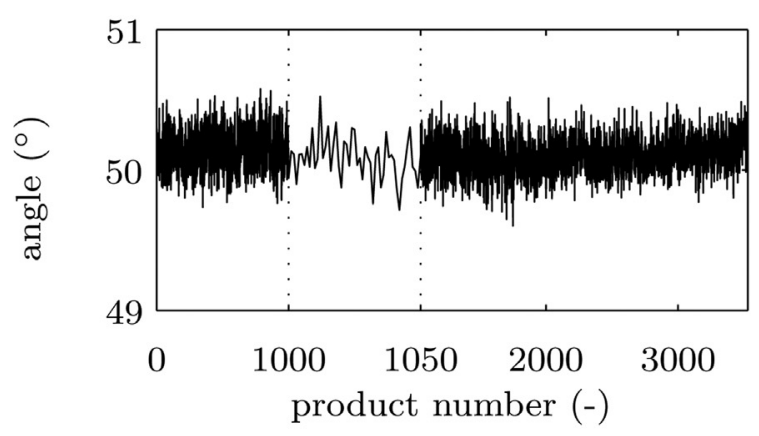

(d)

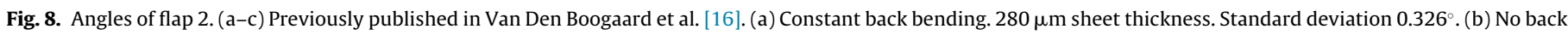

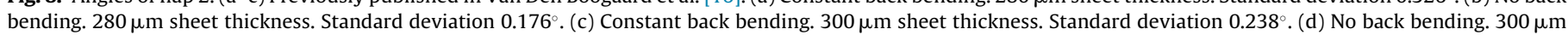
sheet thickness. Standard deviation $0.135^{\circ}$.

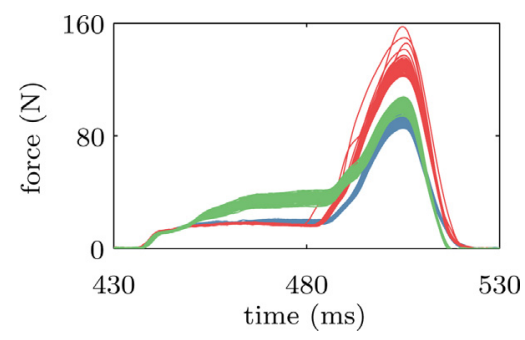

Fig. 9. Bending force of subset from dataset 3 ( $300 \mu \mathrm{m}$ with back bending), for benders one ( $\_$), two (_ $\_$) and three ( $\_$).

imum value just before the deepest point of the press due to strain rate sensitivity of the material. The exact length of the bender strongly influences the maximum bending force. The increase of the force of bender 3 around $450 \mathrm{~ms}$ is caused by contact between the bender and the die due to misalignment of the tooling. Fig. 9 also gives an impression of the amount of variation of the bending forces during the test. In Section 5.5 it will be investigated how these variations relate to the variation of the final angle of the flap.

\subsection{Deepdrawing variation}

The hydraulic system for the blank holders of the deep drawing stage has a strong effect on the stability of the whole process. The stability of the hydraulic system is poor due to several issues, such as the positioning of the valves and sensors, the length of the tubes and the limited size of the oil reservoir. Therefore, the complete system is re-pressurized every few seconds, causing a periodic variation in the blank holder pressure. The system is repressurized to $p_{\max }$ when the pressure drops below $p_{\text {min }}$. Most test runs were performed with a pressure range of 102-130 bar, causing the system to re-pressurize after every eight products. The pressure



(a)

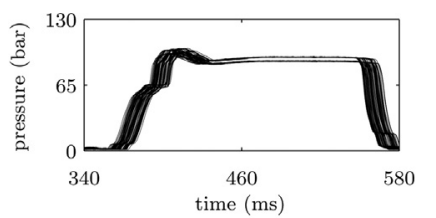

(b)
Fig. 10. Pressure from blank holder 2 for 100 subsequent products with different settings for the hydraulic system. (a) Dataset $2, p_{\min } / p_{\max }=102 / 130$ bar. (b) Dataset $4, p_{\min } / p_{\max }=100 / 105$ bar.

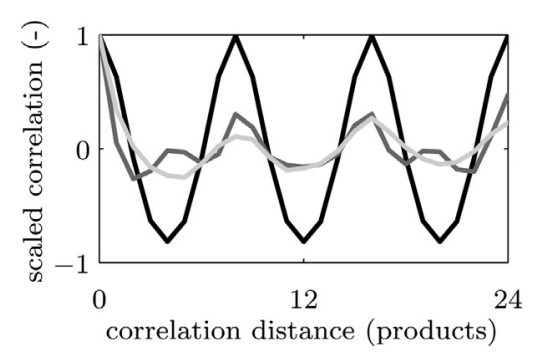

Fig. 11. Autocorrelation of maximum pressure of blank holder 2 ( $\_$), maximum forging force ( $\_$) and maximum force of bender $2\left(\_\right)$for dataset $2(280 \mu \mathrm{m}$ without back bending).

of one blank holder of dataset 2 is shown in Fig. 10a. Less variation was observed in test number 4 due to the narrower range of 100-105 bar, which caused the system to re-pressurizes after every two products (Fig. 10b).

In Fig. 11, the autocorrelation of the blankholder pressure of dataset 2 is shown. The period of 8 products for pressurizing the system can be clearly observed. In the same figure, it can be seen that other process steps are also affected by the variation of the 


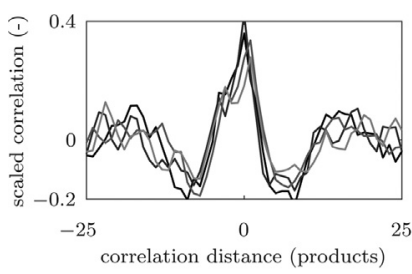

(a)

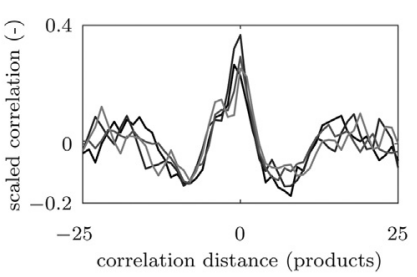

(b)
Fig. 12. Scaled cross-correlation between thickness and forces for all four datasets. (a) Cross-correlation between thickness and maximum cutting force. (b) Crosscorrelation between thickness and maximum force of bender 2 .

hydraulic system: the autocorrelation of the maximum forging force and of the maximum bending force have the same periodicity of 8 products. One cause for the interaction between the process steps is the compliance of the stamping press. Variation of the force at one position in the press affects the deformation of the tooling and consequently affects the other stages of production. This can be verified with the cross-correlation plots between the maximum deep drawing, forging and bending forces.

In Fig. 11, the autocorrelation of the blank holder pressure of dataset 2 is shown. The period of eight products for pressurizing the system can be clearly observed. In the same figure, it can be seen that other process steps are also affected by the variation of the hydraulic system: the autocorrelation of the maximum forging force and of the maximum bending force have the same periodicity of eight products. A cause for the interaction between the process steps is the compliance of the stamping press. Variation of the force at one position in the press affects the deformation of the tooling and consequently affects the other stages of production. This can be verified with the cross-correlation plots between the maximum deep drawing, forging and bending forces [17].

\subsection{Thickness variation}

In the demonstrator process, the thickness of the incoming sheet is measured for every product. Hence, the effect of sheet thickness on the process forces can be investigated. First, the crosscorrelation between the thickness and the maximum cutting force is shown in Fig. 12a for all datasets. As expected, the correlation between thickness and maximum cutting force is positive: a thicker sheet requires a higher cutting force. The cross-correlation between bending force and thickness gives a similar result (Fig. 12b). Hence, part of the variation of the process forces can be attributed to variation of the thickness of the sheet. However, the correlation value of less than 0.4 shows that the effect of the thickness on the cutting and bending is limited with respect to the total variation in the process.

\subsection{Correlation between forces and angle}

The objective of this work is to investigate the use of force measurements for control of the bending stage. To detect the relations between small variations in the process forces and the angle of the flap, the correlations should be identified using large datasets. Two examples of weak but clear correlations between forces and the angle can be found in Fig. 13. These correlations have a value of -0.32 and 0.18 . The size of the datasets enables identification of these correlations with statistical significance: the $p$-values are $2 \cdot 10^{-85}$ and $6 \cdot 10^{-27}$ respectively.

\section{Process estimator}

Several relations in the measurement data have been discussed in Section 4. Although the correlations between measurement data

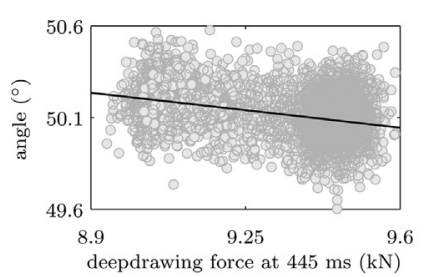

(a)

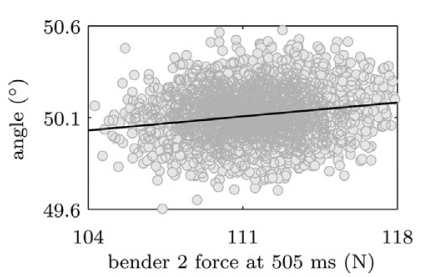

(b)
Fig. 13. Angle versus force data for dataset 4. (a) Angle versus deep drawing force at $445 \mathrm{~ms}$, with correlation value -0.32 . (b) Angle versus bender 2 force at $505 \mathrm{~ms}$ with correlation value 0.18

and the final angle are weak, they can be used in a feedforward estimator. In this section, the effect of feedforward control with force measurements is estimated based on process control simulations which have been performed using experimental data obtained from the demonstrator process. A general linearized model of a metal forming process is given in Section 5.1. Following this model, the equations for fitting a regression model based on historical data are given in Section 5.2, and the regression method used for fitting is discussed in Section 5.3. The assumptions and procedure for simulating process control based on experimentally obtained process data are given in Section 5.4 and the results are presented in Section 5.5 .

\subsection{Process control}

A system diagram for control of metal forming processes is given in Fig. 14. The process may be regarded as a discrete system, where every product is one sample. The process is divided into two parts, where $P^{(1)}$ is the part of the process which provides measurements for feedforward control and $P^{(2)}$ is the part of the process which is affected by the control system. Both parts consist of one or more process stages. The properties of the product after the first part of the process are denoted by $\boldsymbol{y}_{1} \in \mathbb{R}^{y_{1}}$. The control parameters $\boldsymbol{u} \in \mathbb{R}^{u}$ act on the second part of the process. The final properties of the product are denoted with $\boldsymbol{y}_{2} \in \mathbb{R}^{y_{2}}$ and the error in product properties with $\boldsymbol{e} \in \mathbb{R}^{y_{2}}$. The first part of the process delivers a set of measurements $\boldsymbol{m} \in \mathbb{R}^{m}$ to be used in a feedforward loop. The disturbances $\boldsymbol{d}_{1} \in \mathbb{R}^{d_{1}}$ and $\boldsymbol{d}_{2} \in \mathbb{R}^{d_{2}}$ act on both parts of the process $P^{(1)}$ and $P^{(2)}$ respectively.

The sources of variation can be seen as disturbances to the system. Some important sources of variation in metal forming are variation of material properties, sheet thickness, lubrication properties and tool wear. These sources are recognized by many researchers in the field of robust optimization and control of forming processes. An overview of different studies on these sources of variation is given in the work of Hazra et al. [18]. However, there are many other sources of variation which receive less attention in studies on the accuracy of metal forming processes. Col [19] gives an overview of factors affecting forming processes in an industrial environment based on his professional experience. He names a large number of influencing factors such as variation of blankholder forces, guidance of the rams, the effect of temperature on lubrication, localization of lubricant, tooling positioning and press stiffness. In many cases, it is unfeasible to perform the extensive measurements needed to quantify these sources of variation and their effect on the forming process.

Variations in the forming process evolve over time, although different sources of variation may have different dynamics: tool wear increases slowly during lifetime of the tooling, whereas the temperature of the tooling increases rapidly during running-in. For control of forming processes, it is essential to have direct or indirect measurements of the disturbances to the system. In other words, important sources of variation should be observable. 


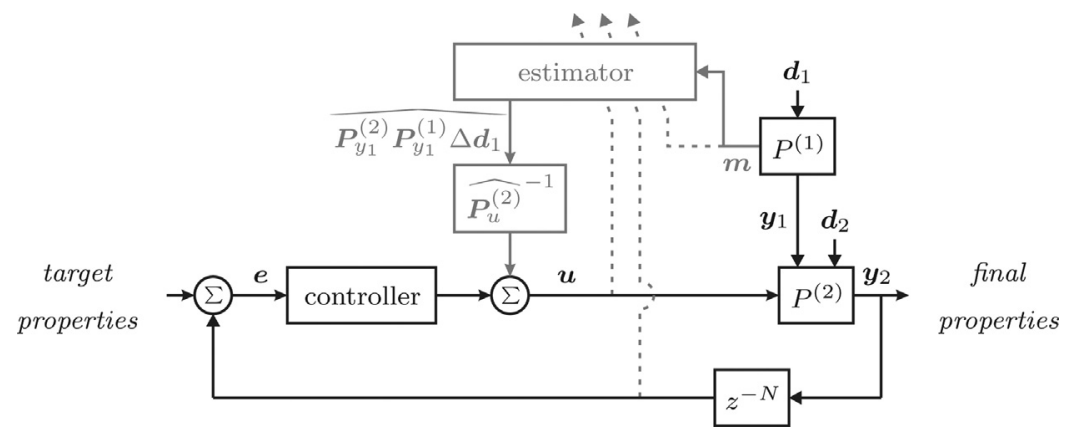

Fig. 14. System diagram for control of a metal forming process. The components of the feedforward system are shown in gray. The hât represents estimated values.

Due to elastic springback, the final state of the product is only reached after release of the product. Consequently, the final state can only be directly measured when the product is finished and no control actions can be applied anymore. In the control diagram this is indicated with the delay block $z^{-N}$, where $N$ is the measurement delay in number of products. The measurement delay is at least one product, but it may be several products, depending on the speed and position of the measurement system.

When compensating for long-term variations in a mass production process, it may be sufficient to measure the final state of a product and compensate the error for the upcoming products with a feedback loop. The feedback loop is shown in black in the control diagram. However, product-to-product variations cannot be eliminated in such an approach. Other measurements should be used to estimate the effect of product-to-product variations on the final state of the product. These may be either direct measurements on the intermediate state of the product, or indirect measurements which carry information about the process and the product, such as force measurements. These measurements can be used in a feedforward loop to control the process.

To determine the relation between the measurements $\boldsymbol{m}$ and the final properties of the product $\boldsymbol{y}_{2}$, the mathematical equations of the process are determined and linearized. Firstly, the process is described with the unknown functions $f, g$, and $h$, which are functions of the disturbances $\boldsymbol{d}_{1}$ and $\boldsymbol{d}_{2}$ and the control parameters $\boldsymbol{u}$ :

$\boldsymbol{m}=f\left(\boldsymbol{d}_{1}\right)$

$\boldsymbol{y}_{1}=g\left(\boldsymbol{d}_{1}\right)$

$\boldsymbol{y}_{2}=h\left(\boldsymbol{y}_{1}, \boldsymbol{d}_{2}, \boldsymbol{u}\right)$

These equations are linearized around their nominal values $\boldsymbol{d}_{1}^{\star}$, $\boldsymbol{d}_{2}^{\star}$ and $\boldsymbol{u}^{\star}$ using Taylor expansion:

$$
\begin{aligned}
\boldsymbol{m} \approx f( & \left.\boldsymbol{d}_{1}^{\star}\right)+\left(\boldsymbol{d}_{1}-\boldsymbol{d}_{1}^{\star}\right) \cdot \nabla f\left(\boldsymbol{d}_{1}^{\star}\right) \\
\boldsymbol{y}_{1} \approx g & \left(\boldsymbol{d}_{1}^{\star}\right)+\left(\boldsymbol{d}_{1}-\boldsymbol{d}_{1}^{\star}\right) \cdot \nabla g\left(\boldsymbol{d}_{1}^{\star}\right) \\
\boldsymbol{y}_{2} \approx & h\left(g\left(\boldsymbol{d}_{1}^{\star}\right), \boldsymbol{d}_{2}^{\star}, \boldsymbol{u}^{\star}\right) \\
& +\left(\left(\boldsymbol{d}_{1}-\boldsymbol{d}_{1}^{\star}\right) \cdot \nabla g\left(\boldsymbol{d}_{1}^{\star}\right)\right) \cdot \nabla_{y_{1}} h\left(g\left(\boldsymbol{d}_{1}^{\star}\right)\right) \\
& +\left(\boldsymbol{d}_{2}-\boldsymbol{d}_{2}^{\star}\right) \cdot \nabla_{d_{2}} h\left(\boldsymbol{d}_{2}^{\star}\right) \\
& +\left(\boldsymbol{u}-\boldsymbol{u}^{\star}\right) \cdot \nabla_{u} h\left(\boldsymbol{u}^{\star}\right)
\end{aligned}
$$

The derivatives in the equations above are renamed to:

$$
\begin{aligned}
& \boldsymbol{P}_{m}^{(1)}=\nabla f\left(\boldsymbol{d}_{1}^{\star}\right) \\
& \boldsymbol{P}_{y_{1}}^{(1)}=\nabla g\left(\boldsymbol{d}_{1}^{\star}\right) \\
& \boldsymbol{P}_{y_{1}}^{(2)}=\nabla_{y_{1}} h\left(g\left(\boldsymbol{d}_{1}^{\star}\right)\right) \\
& \boldsymbol{P}_{d_{2}}^{(2)}=\nabla_{d_{2}} h\left(\boldsymbol{d}_{2}^{\star}\right)
\end{aligned}
$$

$\boldsymbol{P}_{u}^{(2)}=\nabla_{u} h\left(\boldsymbol{u}^{\star}\right)$

The changes of the measurements $\boldsymbol{m}$ and the final product properties $\boldsymbol{y}_{2}$ with respect to their nominal values can now be written as:

$\Delta \boldsymbol{m}=\boldsymbol{P}_{m}^{(1)} \Delta \boldsymbol{d}_{1}$

$\Delta \boldsymbol{y}_{2}=\boldsymbol{P}_{y_{1}}^{(2)} \boldsymbol{P}_{y_{1}}^{(1)} \Delta \boldsymbol{d}_{1}+\boldsymbol{P}_{d_{2}}^{(2)} \Delta \boldsymbol{d}_{2}+\boldsymbol{P}_{u}^{(2)} \Delta \boldsymbol{u}$

The target properties $\boldsymbol{y}_{2}$ are influenced by three components: two are related to the disturbances $\boldsymbol{d}_{1}$ and $\boldsymbol{d}_{2}$, and one is related to the control parameters $\boldsymbol{u}$. The component $\boldsymbol{P}_{d_{2}}^{(2)} \Delta \boldsymbol{d}_{2}$ cannot be estimated in the feedforward loop because there are no measurements available from $P^{(2)}$. Hence, the goal of the feedforward loop is to estimate the component $\boldsymbol{P}_{y_{1}}^{(2)} \boldsymbol{P}_{y_{1}}^{(1)} \Delta \boldsymbol{d}_{1}$ from Eq. (16) based on the measurements $\boldsymbol{m}$. Using Eq. (15) and assuming that $\boldsymbol{P}_{m}^{(1)^{-1}}$ exists, it follows:

$\boldsymbol{P}_{y_{1}}^{(2)} \boldsymbol{P}_{y_{1}}^{(1)} \Delta \boldsymbol{d}_{1}=\boldsymbol{P}_{y_{1}}^{(2)} \boldsymbol{P}_{y_{1}}^{(1)} \boldsymbol{P}_{m}^{(1)^{-1}} \Delta \boldsymbol{m}$

Hence, the relation between the variation in the measurements $\Delta \boldsymbol{m}$ and the variation of the final properties of the product $\Delta \boldsymbol{y}_{2}$ due to the disturbance $\boldsymbol{d}_{1}$ is given by the matrix $\boldsymbol{P}_{y_{1}}^{(2)} \boldsymbol{P}_{y_{1}}^{(1)} \boldsymbol{P}_{m}^{(1)^{-1}}$. This matrix has to be estimated to be able to use feedforward control based on the measurements $\boldsymbol{m}$.

Different approaches may be used to determine the matrix $\boldsymbol{P}_{y_{1}}^{(2)} \boldsymbol{P}_{y_{1}}^{(1)} \boldsymbol{P}_{m}^{(1)^{-1}}$. This may be done either offline (e.g. using a numerical or analytical model) or online based on historical data of the process. The latter option is indicated in the control diagram (Fig. 14) by the dashed lines, indicating which data is required to estimate the relation between the measurements and the final properties of the product.

\subsection{Model fitting}

In this work, it is proposed to use historical data from the manufacturing process to determine the relations between process measurements and final properties of the products. At every moment during production, it is expected that the most recent produced products have the highest resemblance with the currently produced products. Hence, it is preferable to use the most recent data for the process estimator. To do so, a moving window process estimator is proposed, which is continuously updated based on the $n_{p}$ most recently produced products. Two different cases are considered: the case that the matrix $\boldsymbol{P}_{u}^{(2)}$ for the effect of the control parameters on the final product properties is known, and the case that it is unknown. In the latter case, estimation of $\boldsymbol{P}_{u}^{(2)}$ is included 
in the procedure. For the former case, start with combining Eqs. (16) and (15) to:

$\Delta \boldsymbol{y}_{2}-\boldsymbol{P}_{u}^{(2)} \Delta \boldsymbol{u}=\boldsymbol{P}_{y_{1}}^{(2)} \boldsymbol{P}_{y_{1}}^{(1)} \boldsymbol{P}_{m}^{(1)}{ }^{-1} \Delta \boldsymbol{m}+\boldsymbol{P}_{d_{2}}^{(2)} \Delta \boldsymbol{d}_{2}$

In this equation, $\boldsymbol{y}_{2}$ are the final properties of the product, $\boldsymbol{u}$ the control parameters, $\boldsymbol{m}$ the force measurements and $\boldsymbol{d}_{2}$ the unobservable disturbances. The mean values in the dataset with $n_{p}$ products are subtracted from the data to determine the variations. The above equation holds for a single product. Expanding the equation to all $n_{p}$ products gives:

$\Delta \boldsymbol{Y}_{2}-\boldsymbol{P}_{u}^{(2)} \Delta \boldsymbol{U}=\boldsymbol{P}_{y_{1}}^{(2)} \boldsymbol{P}_{y_{1}}^{(1)} \boldsymbol{P}_{m}^{(1)^{-1}} \Delta \boldsymbol{M}+\boldsymbol{P}_{d_{2}}^{(2)} \Delta \boldsymbol{D}_{2}$

The dataset of historical data contains $\Delta \boldsymbol{Y}_{2}, \Delta \boldsymbol{U}$ and $\Delta \boldsymbol{M}$. It is assumed that $\boldsymbol{P}_{d_{2}}^{(2)} \Delta \boldsymbol{D}_{2}$ acts as Gaussian white noise on the system. The system of equations can be rewritten to a regression problem in the form $\boldsymbol{Z}=\boldsymbol{\beta}_{1} \Delta \boldsymbol{M}+\boldsymbol{\epsilon}_{1}$, with:

$\boldsymbol{Z}=\Delta \boldsymbol{Y}_{2}-\boldsymbol{P}_{u}^{(2)} \Delta \boldsymbol{U}$

$\boldsymbol{\beta}_{1}=\boldsymbol{P}_{y_{1}}^{(2)} \boldsymbol{P}_{y_{1}}^{(1)} \boldsymbol{P}_{m}^{(1)^{-1}}$

$\boldsymbol{\epsilon}_{1}=\boldsymbol{P}_{d_{2}}^{(2)} \Delta \boldsymbol{D}_{2}$

Regression methods can be used to determine the coefficient matrix $\boldsymbol{\beta}_{1}$, establishing the process estimator. Thereafter, the process estimator can be used in the control system to interpret new measurements $\boldsymbol{m}$. Note that it is not needed to know the size of the disturbance vector $\boldsymbol{d}_{1}$ to establish the relation between $\boldsymbol{Z}$ and $\boldsymbol{M}$.

In the case that the transfer matrix from control parameter to target properties $\boldsymbol{P}_{u}^{(2)}$ is unknown, Eq. (19) is written as:

$\Delta \boldsymbol{Y}_{2}=\left[\begin{array}{ll}\boldsymbol{P}_{y_{1}}^{(2)} \boldsymbol{P}_{y_{1}}^{(1)} \boldsymbol{P}_{m}^{(1)^{-1}} & \boldsymbol{P}_{u}^{(2)}\end{array}\right]\left[\begin{array}{c}\Delta \boldsymbol{M} \\ \Delta \boldsymbol{U}\end{array}\right]+\boldsymbol{P}_{d_{2}}^{(2)} \Delta \boldsymbol{D}_{2}$

This equation can be rewritten to the form $\Delta \boldsymbol{Y}_{2}=\boldsymbol{\beta}_{2} \boldsymbol{X}+\boldsymbol{\epsilon}_{2}$ and $\boldsymbol{\beta}_{2}$ can be determined, with:

$\boldsymbol{\beta}_{2}=\left[\begin{array}{ll}\boldsymbol{P}_{y_{1}}^{(2)} \boldsymbol{P}_{y_{1}}^{(1)} \boldsymbol{P}_{m}^{(1)} & \boldsymbol{P}_{u}^{(2)}\end{array}\right]$

$\boldsymbol{X}=\left[\begin{array}{c}\Delta \boldsymbol{M} \\ \Delta \boldsymbol{U}\end{array}\right]$

$\boldsymbol{\epsilon}_{2}=\boldsymbol{P}_{d_{2}}^{(2)} \Delta \boldsymbol{D}_{2}$

These equations are based on the following assumptions. Violations of these assumptions may affect the quality of the process estimator.

1. The system is linear in the range of variation of $\boldsymbol{d}_{1}$ and $\boldsymbol{u}$.

2. The inverse mapping $\boldsymbol{P}_{m}^{(1)^{-1}}$ exists. Different disturbances $\boldsymbol{d}_{1}$ result in different measurements $\boldsymbol{m}$.

3. The known transfer matrix $\boldsymbol{P}_{u}^{(2)}$ value is correct.

4. There is enough data available for estimation of the coefficient matrix $\boldsymbol{\beta}_{1}$ or $\boldsymbol{\beta}_{2}$. The required amount of data also depends on the measurement noise and the magnitude of the unobservable disturbances $\boldsymbol{d}_{2}$ with respect to the observable disturbances $\boldsymbol{d}_{1}$.

5. Disturbances $\boldsymbol{d}_{1}$ and $\boldsymbol{d}_{2}$ are uncorrelated. Violation of this assumption implies that the variations of $\boldsymbol{d}_{2}$ can be partly observed in the measurements $\boldsymbol{m}$, leading to an improvement of the feedforward control system.

6. Disturbance $\boldsymbol{d}_{2}$ acts as Gaussian white noise on the system. This may be incorrect because the variation of disturbance $\boldsymbol{d}_{2}$ may be correlated in time or determined by a different probability distribution.
These assumptions do not restrict the fitting procedure of the linear regression model with the dataset of $\boldsymbol{Y}_{2}, \boldsymbol{U}$ and $\boldsymbol{M}$. It is proposed to use the LASSO regression method for fitting the linear model. The LASSO method is discussed in the following section.

\subsection{LASSO regression}

The derivation in this section follows the case that $\boldsymbol{P}_{u}^{(2)}$ is known (Eqs. (19)-(22)), whereas the case that $\boldsymbol{P}_{u}^{(2)}$ is unknown (Eqs. (23)-(26)) can be derived equivalently. In the dataset which is used to fit the model, $n_{p}$ is the number of products, $n_{m}$ the number of measurements per product, $n_{y_{2}}$ the number of output properties per product and $n_{u}$ the number of control parameters. The $n_{y_{2}}$ rows of the output matrix $\boldsymbol{Z}$ from Eq. (20) can be fitted separately. Hence, the system to be solved has the form $\boldsymbol{z}=\boldsymbol{\beta}_{1} \Delta \boldsymbol{M}$, where $\boldsymbol{z}$ has size $\left(1 \times n_{p}\right), \boldsymbol{\beta}_{1}$ has size $\left(1 \times n_{m}\right)$ and $\Delta \boldsymbol{M}$ has size $\left(n_{m} \times n_{p}\right)$.

Every row of the input matrix $\Delta \boldsymbol{M}$ stands for one of the measurements minus the mean value of that measurement over the fitting dataset. For the demonstrator process, these are measurements from one force sensor at different sampling points of the force curves. This is different than what has been done in the studies discussed in Section 1. The usual approach is to extract one or few characteristics from the force curves. However, we propose to use the data from the full force curve to maximize the information gathered during production. Matrix $\Delta \boldsymbol{M}$ is expected to be multicollinear because different measurements are expected to correlate.

When the number of measurements per product is much smaller than the number of products in the dataset $\left(n_{m} \ll n_{p}\right)$, the coefficients $\boldsymbol{\beta}_{1}$ may be solved with the Ordinary Least Squares (OLS) method. However, when using data from force measurements in a process estimator, $n_{m}$ may easily exceed $n_{p}$. In that case, no unique solution can be found with OLS. Hence, other regression methods should be used to find a solution without overfitting the data. Several methods have been developed for fitting data with more independent variables than data points, such as stepwise regression methods, where a subset of the data is selected to fit the model. Other methods, such as ridge regression, LASSO regression [20] and least angle regression [21], restrict the magnitude of the coefficient matrix $\boldsymbol{\beta}_{1}$ to prevent overfitting.

In this work, the LASSO regression method is used for fitting the process estimator. The first step of the fitting procedure is normalization of the data. The output vector $\boldsymbol{z}$ and every column of the input matrix $\Delta \boldsymbol{M}$ is normalized in such a way that its mean becomes zero and its standard deviation becomes one. Thereafter, the coefficient vector $\boldsymbol{\beta}_{1}$ is found based on the following minimization:

$\min _{\boldsymbol{\beta}_{1} \in \mathbb{R}^{m}}\left(\frac{1}{2 n_{p}} \sum_{i=1}^{n_{p}}\left(z_{i}-\boldsymbol{\beta}_{1} \Delta \boldsymbol{m}_{i}\right)^{2}+\lambda \sum_{j=1}^{n_{m}}\left|\beta_{1 j}\right|\right)$

The parameter $\lambda$ restricts the magnitude of the coefficients $\boldsymbol{\beta}_{1}$. If $\lambda$ is zero, Eq. (27) reduces to OLS. Eq. (27) is a convex problem and can be solved with standard optimization techniques. An efficient solver for the LASSO method has been developed by Efron et al. [21]. After fitting the regression model, it can be used to predict the variation in the output properties $\Delta \boldsymbol{y}_{2}$ of a new product given its measurement $\boldsymbol{m}$. For the demonstrator process, the force measurement is used to predict the variation in the final angle of the flap.

\subsection{Simulation of control with force measurements}

In order to estimate the effectiveness of a control system for the bending stage of the demonstrator process, it is essential to use experimental data which is obtained from the demonstrator 


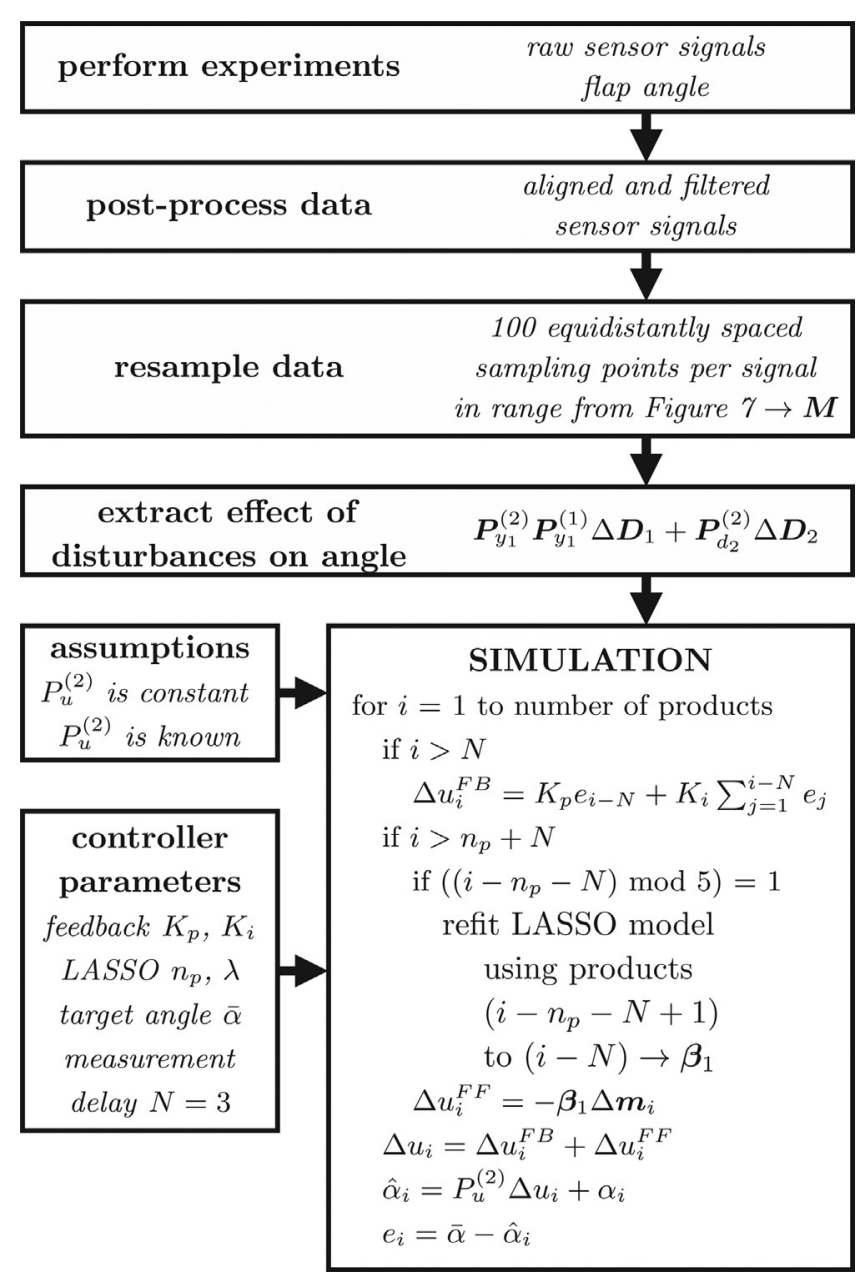

Fig. 15. Simulation flowchart.

process itself. Therefore, a simulation is performed which uses all relevant experimental data from a test (i.e. variations in process forces as well as angle measurements) to estimate how different controllers would have performed if they would have been used for that specific test. The only assumptions which have been made in the simulations are related to the actuation system. It is assumed that the actuation system is perfectly linear and perfectly known. Obviously, the exact performance of the real actuation system (adjustment of the back bending punch position in stage 18 , see Fig. 5b) should be investigated in order to define the exact controller performance. An overview of the simulation procedure is given in Fig. 15. The simulations have been performed using MATLAB.

It must be noted that the traditional approach for validation, where the data is split in a fitting and a validation fraction, cannot be applied with the proposed approach. The moving window process estimator implies that the relation between measurements and final properties of the product is estimated based on the data from the $n_{p}$ most recently produced products. Hence, the change in final product properties of a single product is predicted with a model which never includes data from that product itself. Therefore, the essential condition that data should not be used for fitting and prediction simultaneously is not violated within these simulation runs.

The first steps of the simulation are related to post-processing of the measured data from the demonstrator process. Data from the test runs with back bending are used for the simulation (datasets 1 and 3 from Table 3). The sensor data is aligned and filtered [14] and thereafter resampled. Each sensor signal is resampled within the range shown in Fig. 7 to a grid of 100 equidistant points. Linear interpolation is used if a new sampling point falls in between two original sampling points.

Thereafter, the effect of the disturbances on the angle is extracted from the data. The control parameter $u$ (the back bending end distance) was kept constant during the test runs. Hence, the variation of the angle in the datasets was only caused by the disturbances $\boldsymbol{d}_{1}$ and $\boldsymbol{d}_{2}$. The effect of the disturbances on the final angle during the tests was:

$\boldsymbol{P}_{y_{1}}^{(2)} \boldsymbol{P}_{y_{1}}^{(1)} \Delta \boldsymbol{D}_{1}+\boldsymbol{P}_{d_{2}}^{(2)} \Delta \boldsymbol{D}_{2}=(\boldsymbol{\alpha}-\bar{\alpha})^{T}$

where $\boldsymbol{\alpha}$ is a vector with all measured angles and $\bar{\alpha}$ is the average angle in the dataset.

The disturbances from Eq. (28) are transferred to simulation runs of the control system. Furthermore, it is assumed that the effect of the control parameter on the angle $P_{u}^{(2)}$ is constant (i.e. independent of $u, \boldsymbol{d}_{1}$ and $\boldsymbol{d}_{2}$ ) and perfectly known. Another assumption is that the control system if fast enough to adjust the control parameter $u$ to any position within one stroke of the press. These are the only assumptions needed to determine the effect of a control system on the accuracy of the process. The exact value of $P_{u}^{(2)}$ is not relevant for the simulations, because the effect of $P_{u}^{(2)}$ can be compensated with scaling of the controller parameters. In other words, simulations with different values for $P_{u}^{(2)}$ can give exactly equal results if the controller parameters are scaled accordingly. Therefore, a value of 1 is taken for $P_{u}^{(2)}$. The process equations for the simulation runs can be found by rewriting Eq. (9) using Eq. (28) to:

$$
\begin{aligned}
\hat{\alpha}_{i}=\alpha\left(u^{\star}, \boldsymbol{d}_{1}^{\star}, \boldsymbol{d}_{2}^{\star}\right)+P_{u}^{(2)} \Delta u_{i}+\boldsymbol{P}_{y_{1}}^{(2)} \boldsymbol{P}_{y_{1}}^{(1)} \Delta \boldsymbol{d}_{1} & +\boldsymbol{P}_{d_{2}}^{(2)} \Delta \boldsymbol{d}_{2}= \\
& \bar{\alpha}+P_{u}^{(2)} \Delta u_{i}+\left(\alpha_{i}-\bar{\alpha}\right)
\end{aligned}
$$

where $\hat{\alpha}_{i}$ is the predicted angle with control, and $\alpha_{i}$ is the measured angle from the real test. The control parameter $\Delta u_{i}$ is determined with the control system and has a feedback component $\Delta u_{i}^{F B}$ as well as a feedforward component $\Delta u_{i}^{F F}$. For the feedback loop, a PI controller is used with the following formulation:

$\Delta u_{i}^{F B}=K_{p} e_{i-N}+K_{i} \sum_{j=1}^{i-N} e_{j}$

The measurement delay $N$ is three products. The gain factors for the proportional and integral action are $K_{p}$ and $K_{i}$ respectively. The error of the $i$-th product is denoted by $e_{i}$, with $\bar{\alpha}$ as target angle.

For the feedforward loop, the process estimator is fit based on the dataset of most recent $n_{p}$ products following the approach discussed in Section 5.2. The force measurements are gathered in the measurement matrix $\boldsymbol{M}$. For product $i$, the measurement vector $\boldsymbol{m}_{i}$ contains its force measurements from all process stages. The process estimator is continuously updated after every five products and the feedforward component $\Delta u_{i}^{F F}$ is determined for each product using the LASSO regression model and the force measurements $\boldsymbol{m}_{i}$ from that specific product. If $n_{p}$ is chosen too low, the dataset size is not sufficient to detect the correlations between the measurements and the final angle. On the other hand, only data which is relevant for the current state of the process should be included in the model. Some of the disturbances in $\boldsymbol{d}_{1}$ and $\boldsymbol{d}_{2}$ may change slowly over time. Hence, it is expected that recent data has more resemblance with the current state of the process. Therefore, it is expected that the quality of the model will decrease if $n_{p}$ is chosen too large.

To perform a simulation run, four parameters have to be chosen: the dataset size $n_{p}$, the LASSO parameter $\lambda$ (Eq. (27)) and the gain factors $K_{p}$ and $K_{i}$. Different dataset sizes $n_{p}$ have been used, varying from 100 to 1000 . The $\lambda$ parameter has been varied in between 0.005 and 0.5 . For every combination of $n_{p}$ and $\lambda$, the gain factors 


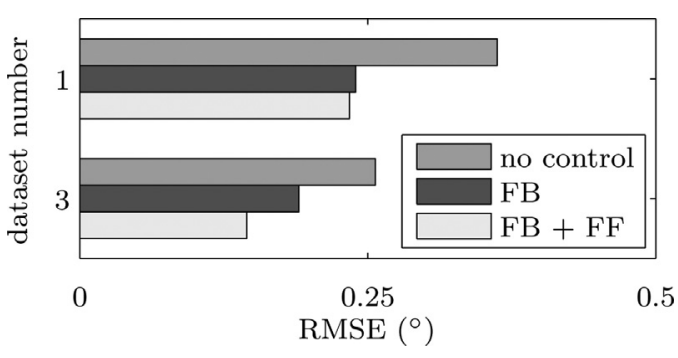

Fig. 16. RMSE of the angle in the simulation runs for all datasets, without control, with feedback (FB) and with feedback and feedforward (FB + FF).

$K_{p}$ and $K_{i}$ have been optimized based on the RMSE of the result. Obviously, this is an unfeasible approach for implementation in a production line, because the RMSE can only be determined afterwards. Therefore, further study is needed to develop a procedure for selection of the gain factors and the model parameters $n_{p}$ and $\lambda$. It is expected that these parameters can be selected and updated online with a learning algorithm.

\subsection{Results}

The procedure from the previous section is applied to the two datasets with constant back bending. The RMSE for the run without control, the simulation run with feedback control and the best simulation run with feedforward control based on force measurements is given in Table 4 and visualized in Fig. 16. The first 1002 products are not used in the RMSE calculation because the feedforward loop is activated at product 1003 in the case with the largest dataset size $n_{p}$. For the runs with control, the gain factors of the PI controller have been optimized. A significant reduction of the RMSE is achieved with feedback control: $34 \%$ and $26 \%$ for datasets 1 and 3 respectively. That is expected, because the long-term variation in these datasets is strong, as can be seen in Fig. 8a and c.

The effect of feedforward control based on force measurements is very different for the two datasets. For dataset 1 with a sheet thickness of $280 \mu \mathrm{m}$, the reduction of RMSE with respect to the run with feedback control is only $2 \%$. The improvement for the run with a sheet thickness of $300 \mu \mathrm{m}$ (dataset 3 ) is $24 \%$. That indicates that the force measurements carry information which can be used to control the process. However, insufficient correlation between the force measurements and the flap angle could be found for dataset 1 $(280 \mu \mathrm{m})$ to be able to reach a significant improvement with feedforward control. A possible cause is that the tooling is designed for a sheet thickness of $300 \mu \mathrm{m}$, and using a nominal sheet thickness of $280 \mu \mathrm{m}$ leads to additional instability of the process and a weaker correlation between process forces and final geometry.

To illustrate the effect of the process estimator on the process accuracy, a zoom of the results of dataset 3 is shown in Fig. 17. Around product 2810, a severe drop in the angle is observed in the dataset without control. The feedback controller reacts to the disturbance with a delay. Around product 2840, the angle returns to the original value, causing an overshoot in the response of the feedback controller. In contrast, the feedforward controller is able to interpret the changes in the process forces and predicts part of the variations in the final angle. When the drop in the angle occurs around product 2810 , the improvement due to the feedforward estimator is weak. However, due to the moving window for fitting the process estimator, this data is added to the model, increasing the predictive ability of the model. When the angle of the uncontrolled run returns to the original value around product 2840 , the feedforward estimator is able to predict this based on the force measurements, preventing the overshoot which occurs if only feedback control is used. Several products later, around product 2885 , the angle suddenly drops for two subsequent products.

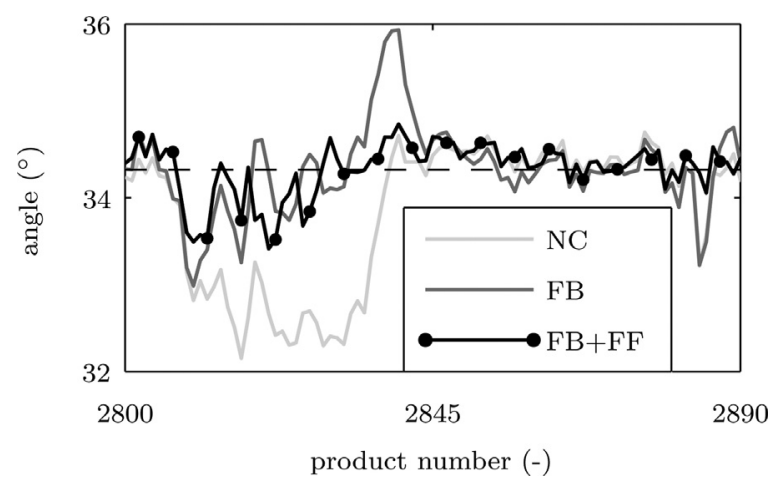

Fig. 17. Zoom of results of dataset $3(300 \mu \mathrm{m})$. The black dashed line indicates the target angle. The process estimator is updated after every product indicated with a black marker.
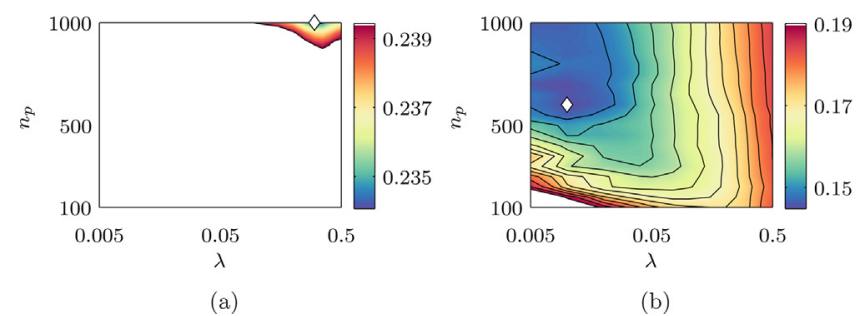

Fig. 18. RMSE of simulation runs with feedforward and optimized PI controller as a function of model parameters.

The feedforward estimator predicts the drop in the angle based on the force measurement and compensates for it.

In Fig. 18, the RMSE of the simulation runs with different model parameters $n_{p}$ and $\lambda$ and optimized PI controller are shown. Only the part of the parameter space which gives an improvement with respect to the run with only feedback control is shown. The best parameter set is marked with a white diamond. For dataset 1 (Fig. 18a), only a small region of the parameter space gives an improvement with respect to feedback control. A small improvement with respect to only feedback control is achieved with a process estimator which is fitted with a large dataset $\left(n_{p}=1000\right)$ and with a strong constraint on the regression parameters $(\lambda=0.3)$.

In contrast to the results from dataset 1 , it can be seen that a large part of the parameter space results in an improvement in accuracy for dataset 3 (Fig. 18b). Hence, the sensitivity of the feedforward system to the model parameters $n_{p}$ and $\lambda$ is not strong. That is important, because these parameters have to be chosen before running the control system on a real production process. The best results are found with $n_{p}=600$ and $\lambda=0.01$. It is noticeable that the best results are not found with the maximum value for the dataset size $\left(n_{p}=1000\right)$. It is expected that recent data has more resemblance with the current state of the process, leading to a decrease in accuracy of the process estimator if too old products are included in the model fitting. However, the accuracy decrease with increasing dataset size is small and no clear conclusions can be drawn based on the current results.

The scrap rate results for dataset 3 are shown in Fig. 19. The percentage of products that do not meet the specifications is shown as a function of the angle specification. A tight angle specification results in high process scrap rate. As observed in Figs. $8 \mathrm{c}$ and 17, the maximum errors may be well over $2^{\circ}$ for short periods during production. However, the most significant improvements of the control systems are reached for angle specifications under $0.5^{\circ}$. The improvement due to feedback control with respect to no control is mainly visible in the decrease of the scrap rate at large values of the angle specification. On the other hand, the scrap rate for the 
Table 4

RMSE of the angle in the simulation runs for all datasets, without control (NC), with feedback (FB) and with feedback and feedforward (FB + FF).

\begin{tabular}{|c|c|c|c|c|c|c|c|}
\hline \multirow[t]{2}{*}{ Dataset number } & \multirow[t]{2}{*}{ Sheet thickness } & \multicolumn{3}{|l|}{ RMSE } & \multicolumn{3}{|c|}{ RMSE reduction } \\
\hline & & $\mathrm{NC}$ & FB & $\mathrm{FB}+\mathrm{FF}$ & $\begin{array}{l}\mathrm{NC} \\
\downarrow \\
\mathrm{FB}\end{array}$ & $\begin{array}{l}\mathrm{FB} \\
\downarrow \\
\mathrm{FB}+\mathrm{FF}\end{array}$ & $\begin{array}{l}\mathrm{NC} \\
\downarrow \\
\mathrm{FB}+\mathrm{FF}\end{array}$ \\
\hline 1 & $280 \mu \mathrm{m}$ & $0.362^{\circ}$ & $0.239^{\circ}$ & $0.234^{\circ}$ & $34 \%$ & $2 \%$ & $35 \%$ \\
\hline 3 & $300 \mu \mathrm{m}$ & $0.256^{\circ}$ & $0.190^{\circ}$ & $0.145^{\circ}$ & $26 \%$ & $24 \%$ & $44 \%$ \\
\hline
\end{tabular}

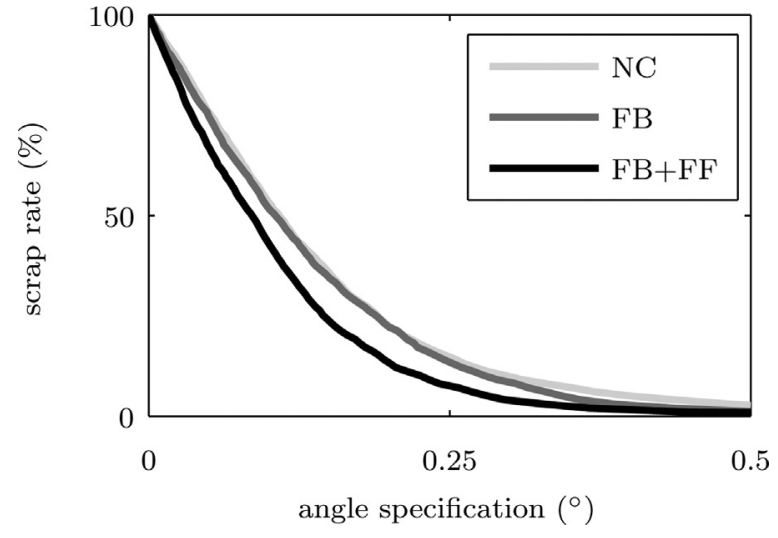

Fig. 19. Scrap rate plot for dataset $3(300 \mu \mathrm{m})$.

feedforward control run is significantly lower than the scrap rate for the feedback control run for all values of the angle specification. The relevance of the use of a feedforward control system depends on the actual process specification. For example, if the process specification is $0.3^{\circ}$, the scrap rates without control, with feedback control and with combined feedforward and feedback control are estimated to be $9.7 \%, 8.4 \%$ and $3.8 \%$ respectively. In that case, the use of feedforward control reduces the scrap rate with more than half with respect to the use of feedback control only.

\section{Conclusion}

In this work, large experimental datasets from an industrial demonstrator process have been used to investigate the feasibility of control of a sheet bending process based on force measurements. The demonstrator process has cutting, deep drawing, forging and bending stages. In Section 4, it was shown that weak correlations can be found between the force measurements and the final angle of one of the flaps of the demonstrator product. Based on these observations, a procedure is proposed for implementing feedforward control with an estimator which uses force measurements to predict the effect of the process disturbances on the final angle of the flap. The LASSO regression method is used to fit process models based on experimental data with more model parameters than datapoints. A moving window is used for fitting the regression model based on the set of $n_{p}$ most recently produced products. The proposed controller has a feedback loop with PI control. The effect of the proposed approach is estimated based on simulation runs. The angle and force measurements of the experimental datasets are used as input for the simulation runs. Based on few assumptions, this data is used to mimic a production process with a control system. For the dataset with a nominal sheet thickness of $300 \mu \mathrm{m}$, a reduction of the RMSE of $24 \%$ is estimated due to the feedforward loop. Hence, it is shown that force measurements carry valuable information which can be used to increase the accuracy of metal forming processes and control the effect of short term variations in the process.

The use of force measurements in control is one of the paths towards more accurate metal forming processes. Feedforward con- trol is needed to eliminate product-to-product variation. Better understanding of the variations in metal forming and their effect on the product and the process forces is needed to take better advantage of the information which is hidden in the force measurements. We foresee that further steps may be taken through the development of self-learning control systems for metal forming, leading to robust control while taking maximum advantage of the data which becomes available during production.

\section{Acknowledgements}

The work leading to these results has received funding from the European Community's Seventh Framework Programme under grant agreement no FP7-285030. We thank the MEGaFiT team for all the contributions which have led to these results.

\section{References}

[1] Allwood J, Duncan S, Cao J, Groche P, Hirt G, Kinsey B, Kuboki T, Liewald M, Sterzing A, Tekkaya A. Closed-loop control of product properties in metal forming. CIRP Ann. Manuf. Technol 2016;65(2):573-96.

[2] Polyblank J, Allwood J, Duncan S. Closed-loop control of product properties in metal forming: a review and prospectus. J. Mater. Process. Technol 2014;214(11):2333-48.

[3] MEGaFiT project, http://cordis.europa.eu/result/rcn/141323_en.html (accessed 01.10.17), 2014.

[4] Hardt D, Hale M, Cook N. Closed loop control of a roll straightening process. CIRP Ann. Manuf. Technol 1984;33(1):137-40.

[5] Müller-Duysing M. Die Berechnung und adaptive Steuerung des Drei-PunktBiegens, Diss. Techn. Wiss. ETH Zürich, Nr. 9989; 1993.

[6] Yang M, Kojima N, Manabe K-I, Nishimura H. High accuracy V-bending process control with an on-line database and modified fuzzy models. JSME Int. J. Ser. C: Dyn. Control Robot. Des. Manuf 1997;40(1):157-62.

[7] Forcellese A, Gabrielli F, Ruffini R. Effect of the training set size on springback control by neural network in an air bending process. J. Mater. Process. Technol 1998;80-81:493-500.

[8] Nastran M, Kuzman K. Stabilisation of mechanical properties of the wire by roller straightening. J. Mater. Process. Technol 2002;125-126:711-9.

[9] Viswanathan V, Kinsey B, Cao J. Experimental implementation of neural network springback control for sheet metal forming. J. Eng. Mater. Technol. Trans. ASME 2003;125(2):141-7.

[10] Heller B, Chatti S, Ridane N, Kleiner M. Online-process control of air bending for thin and thick sheet metal. J. Mech. Behav. Mater 2004;15(6):455-62.

[11] Longo M, Maccarini G. Control system algorithm for the prediction of springback in air bending. Key Eng. Mater 2013;554-557:1382-7.

[12] Groche P, Hoppe F, Hesse D, Calmano S. Blanking-bending process chain with disturbance feed-forward and closed-loop control. J. Manuf. Process 2016;24(1):62-70.

[13] Stellin T, van Tijum R, Engel U. Modelling and experimental study of a microforging process from metal strip for the reduction of defects in mass production. Prod. Eng 2016;10(2):103-12.

[14] Havinga G. Optimization and control of metal forming processes (Ph.D. thesis). University of Twente; 2016.

[15] Nikolić D, Mureşan R, Feng W, Singer W. Scaled correlation analysis: a better way to compute a cross-correlogram. Eur. J. Neurosci 2012;35(5):742-62.

[16] Van Den Boogaard T, Havinga J, Van Tijum R. Model-based control of strip bending in mass production. CIRP Ann. Manuf. Technol 2015;64(1):297-300.

[17] Havinga J, van den Boogaard T. The effect of tooling deformation on process control in multistage metal forming. AIP Conf. Proc 2016;1769:060010.

[18] Hazra S, Williams D, Roy R, Aylmore R, Smith A. Effect of material and process variability on the formability of aluminium alloys. J. Mater. Process. Technol 2011;211(9):1516-26.

[19] Col A. Investigation on press forming scatter origin. Sixth ESAFORM conference on material forming 2003:183-6.

[20] Tibshirani R. Regression shrinkage and selection via the lasso. J. R. Stat. Soc. Ser. B 1996;58(1):267-88.

[21] Efron B, Hastie T, Johnstone I, Tibshirani R. Least angle regression. Ann. Stat 2004;32(2):407-99. 